Research Article

\title{
On the structure of graphs with integer Sombor indices
}

\author{
Tomislav Došlićí ${ }^{1,2, *}$, Tamás Réti ${ }^{3}, \mathrm{Akbar} \mathrm{Ali}^{4}$ \\ ${ }^{1}$ Faculty of Civil Engineering, University of Zagreb, Zagreb, Croatia \\ ${ }^{2}$ Faculty of Information Studies, Novo Mesto, Slovenia \\ ${ }^{3}$ Óbuda University, Bécsiút, Budapest, Hungary \\ ${ }^{4}$ Department of Mathematics, Faculty of Science, University of Hail, Hail, Saudi Arabia
}

(Received: 17 February 2021. Received in revised form: 22 March 2021. Accepted: 26 March 2021. Published online: 1 April 2021.)

(C) 2021 the authors. This is an open access article under the CC BY (International 4.0) license (www.creativecommons.org/licenses/by/4.0/).

\begin{abstract}
The Sombor index is a recently introduced graph-theoretical invariant of the bond-additive type. It is known that it takes integer values for bipartite semi-regular graphs whose degrees appear as two smaller elements in a Pythagorean triple. In this note, we show that it can have integer values also for graphs with more complicated structure and construct infinite families of graphs with integer Sombor indices.
\end{abstract}

Keywords: Sombor index; bond-additive indices; Pythagorean triple; Euler brick.

2020 Mathematics Subject Classification: 05C07, 05C09.

\section{Introduction}

Many graph-theoretical invariants are obtained by adding edge contributions of the type $\varphi(e)=f(x, y)$, where $x$ and $y$ are some numerical characteristics of the end-vertices $u$ and $v$ of an edge $e$ of a graph. Here, $x$ and $y$ are usually taken to be the degrees $d_{u}$ and $d_{v}$ of $u$ and $v$, respectively, but they can also be their eccentricities, transmissions, or any other numerical quantities. Many different functions $f(x, y)$ have been considered so far, giving rise to a variety of indices; the best known among them being the two Zagreb indices, the Randić index, and the ABC index [10]. Curiously enough, the function $f\left(d_{u}, d_{v}\right)=\sqrt{d_{u}^{2}+d_{v}^{2}}$ has been considered only recently in a paper by Ivan Gutman [11] giving rise to a new graph invariant with the name Sombor index.

The new invariant struck a chord with the research community, as witnessed by the stream of recent papers devoted to its properties, generalizations and applicability $[2,4,5,7,9,12,15,18,19,21-25,28,29,32,34,35]$. In the previous paper by the present authors [29], a question was raised about the structure of graphs with integer Sombor indices. It was shown that the only chemical graphs with integral Sombor indices are bipartite semi-regular graphs with degrees 3 and 4 . It was also shown that Sombor indices are integers for all bipartite semi-regular graphs whose degrees $\delta$ and $\Delta$ appear as two smaller elements in a Pythagorean triple, i.e., for which $\sqrt{\delta^{2}+\Delta^{2}} \in \mathbb{N}$. The existence of non-bipartite graphs with integer Sombor indices was left unanswered. In this note, we show that such graphs exist and we construct infinite families of such graphs.

\section{Definitions and preliminary results}

All graphs considered in this note are simple and finite. Notation and terminology from graph theory, used but not defined here, can be found in any of standard graph-theory monographs [3,6]. We denote the vertex set and the edge set of a graph $G$ by $V(G)$ and $E(G)$, respectively. An edge $e \in E(G)$ connecting the vertices $u$ and $v$ is denoted by $u v$. The set of all different degrees of the vertices of a graph $G$ is known as the degree set of $G$. A graph whose degree set consists of one/two/three element(s) is called regular/bidegreed/tridegreed graph, respectively. A graph is bipartite semi-regular if it is bipartite bidegreed and all vertices in the same class of bipartition have the same degree. In such graphs all edges connect vertices of different degrees. In this note, we are interested exclusively in connected graphs.

A bond-additive invariant of a graph $G$ is any invariant $I(G)$ obtained as the sum of edge contributions. Hence,

$$
I(G)=\sum_{u v \in E(G)} \varphi(u v)
$$


where $\varphi(u v)$ is a suitably defined function of some numerical properties of the edge $u v$ and/or of its end-vertices. Some of the best known and most researched topological indices belong to this class - the Randic index [20,26], the Szeged index [13], the Albertson irregularity index [1], and, in case of trees, also the Wiener index [33]. (See also [16] for edge contributions to Wiener index of graphs with cycles.) Among the more recent ones, we mention various connectivity indices, the $\mathrm{ABC}$ index [14] and the Mostar index [8]. We refer the reader to [31] for more detail on bond-additive indices.

The Sombor index of a graph $G$ is defined [11] as

$$
\mathrm{SO}(G)=\sum_{u v \in E(G)} \sqrt{d_{u}^{2}+d_{v}^{2}}
$$

where $d_{u}$ and $d_{v}$ are the degrees of the vertices $u$ and $v$, respectively. Unlike many other bond-additive invariants, the Sombor index is generally not an integer.

It is clear from the definition that the an edge contribution $\varphi(u v)=\sqrt{d_{u}^{2}+d_{v}^{2}}$ is an integer if and only if it forms a Pythagorean triple $\left(d_{u}, d_{v}, \varphi(u v)\right)$ with the degrees of $u$ and $v$. Since the square root of a non-negative integer is either irrational or an integer, the Sombor index of a graph $G$ can be an integer if and only if all edge contributions are integers. This follows from the fact that the number of contributions is itself an integer, $|E(G)|$. The simplest case, when all contributions are integers and equal, leads to semi-regular bipartite graphs. The smallest example is the complete bipartite graph $K_{3,4}$ whose Sombor index is equal to 60 . Another simple example is the graph of the rhombic dodecahedron, a bipartite semi-regular polyhedral graph with 14 vertices (6 of degree 4 and 8 of degree 3), 24 edges and 12 rhombic faces. Its Sombor index is equal to 120 . Generally, the Sombor index of a bipartite semi-regular graph $G_{\Delta, \delta}$ with degrees $(\Delta, \delta)$ is given by $\mathrm{SO}\left(G_{\Delta, \delta}\right)=|E(G)| \sqrt{\Delta^{2}+\delta^{2}}$.

If an edge $u v$ connects two vertices of the same degree $d$, then its contribution $\varphi(u v)=d \sqrt{2}$ cannot be an integer. From there it follows that there cannot exist edges between vertices of the same degree if the graph under consideration has integer Sombor index. In other words, in a graph with integer Sombor index vertices of the same degree must form independent sets. That enables us to set the case of connected bipartite graphs.

Proposition 2.1. Let $G$ be a connected bipartite graph. Then $\mathrm{SO}(G)$ is an integer if and only if $G$ is bipartite semi-regular and its degrees $\delta$ and $\Delta$ appear as non-maximal elements in some Pythagorean triple.

In our previous paper [29], we suggested that all graphs with integer Sombor indices are bipartite. In the following section, we show that this is not the case.

\section{Building graphs from Euler bricks}

We start with the following simple observation.

Lemma 3.1. Let $G$ be a non-bipartite graph with integer Sombor index. Then it must contain vertices of at least three different degrees.

Proof. Since $G$ is not bipartite, there must be at least one odd cycle $C_{k}$ in $G$. If there are only two possible degrees of vertices of $G$, then at least one edge $e$ of $C_{k}$ connects two vertices of the same degree. But then its contribution, $\varphi(e)$, is irrational, and hence $\mathrm{SO}(G)$ cannot be an integer, a contradiction.

Hence, our hypothetic non-bipartite graphs with integer Sombor indices must have at least three different degrees. Denote them by $a, b, c$. We first look at non-bipartite tridegreed graphs with degrees $a, b$, and $c$. In such graphs, the quantities $m_{a, b}, m_{a, c}$ and $m_{b, c}$ must be non-zero, where $m_{i, j}$ is the number of edges connecting the vertices of degrees $i$ and $j$ in a graph. Integrality of contributions implies that the sum of squares of any two of them must be a square itself:

$$
a^{2}+b^{2}=p^{2}, \quad b^{2}+c^{2}=q^{2}, \quad c^{2}+a^{2}=r^{2} .
$$

The first question is: whether such triples exist? The second one is: can they be used to construct graphs with integer Sombor indices?

A quick brute-force search for such triples returned two triples, $(44,117,240)$ and $(85,132,720)$, and checking them in the On-Line encyclopedia of Integer Sequences [30] revealed their belonging to a well-known family of Euler bricks. Namely, in geometrical terms, one can think of $a, b$, and $c$ as the sides of a rectangular prism (a brick) with all face diagonals integer. Such bricks have been known under the name of Euler bricks. It is an open problem whether the body diagonal of an Euler brick can be an integer. This problem is known as the perfect cuboid problem. The literature on Euler bricks and perfect cuboid problem is vast; we refer the reader to $[17,27]$ and references therein for more information on their history and recent developments. For our purpose, it suffices to know that the smallest Euler brick has the sides of length 44, 117, and 240 , and this is the brick we use to construct our desired graphs. 
Theorem 3.1. There are infinitely many non-bipartite graphs with integer Sombor indices. The smallest of them has 358 vertices and 28102 edges.

Proof. We construct a non-bipartite graph $G_{m}$ with integer Sombor index as follows. Take the complete bipartite graph $K_{117,240}$ with classes of bipartition $A$ and $B$ of sizes 117 and 240, respectively, and remove edges of a matching of size 22. That leaves 22 vertices of degree 239 in the smaller class of bipartition and 22 vertices of degree 116 in the larger class. Denote them by $u_{1}, \ldots, u_{22}$ and $v_{1}, \ldots, v_{22}$, respectively. Take an additional vertex $w$ and connect it to all $u_{i}$ and $v_{i}$ for $i=1, \ldots, 22$. The new vertex is of degree 44 and all $u_{i}, v_{i}$ have their degrees restored to 117 and 240, respectively. But, the triple $(44,117,240)$ contains the sides of an Euler brick, in fact the smallest one, found by Halcke in 1719. Hence, the sums of squares of any two of them are squares, and all contributions to Sombor index of $G_{m}$ are integers. Since $(44,117,240)$ is the smallest Euler brick, $G_{m}$ is the smallest non-bipartite graph with integer Sombor index. It has 358 vertices and $117 \cdot 240-22+44=28102$ edges.

It remains to show that there are infinitely many such graphs. It follows from the fact that if $(a, b, c)$ are sides of an Euler brick, then the triple $(k a, k b, k b)$ constitutes another Euler brick for any non-negative integer $k$. Moreover, for our initial $(44,117,240)$ triple, the smallest element in all new triples will remain even, enabling the repetition of our construction.

The constructed graph $G_{m}$ is shown in Figure 1. The removed edges are shown in dashed lines, and all other edges between $A$ and $B$ are omitted for clarity. The degrees of vertices for each class are shown, while the notation $C$ is suppressed. The constructed graph is tripartite and tridegreed, with classes $A, B, C$ of sizes 117, 240, and 1, with degrees 240 ,

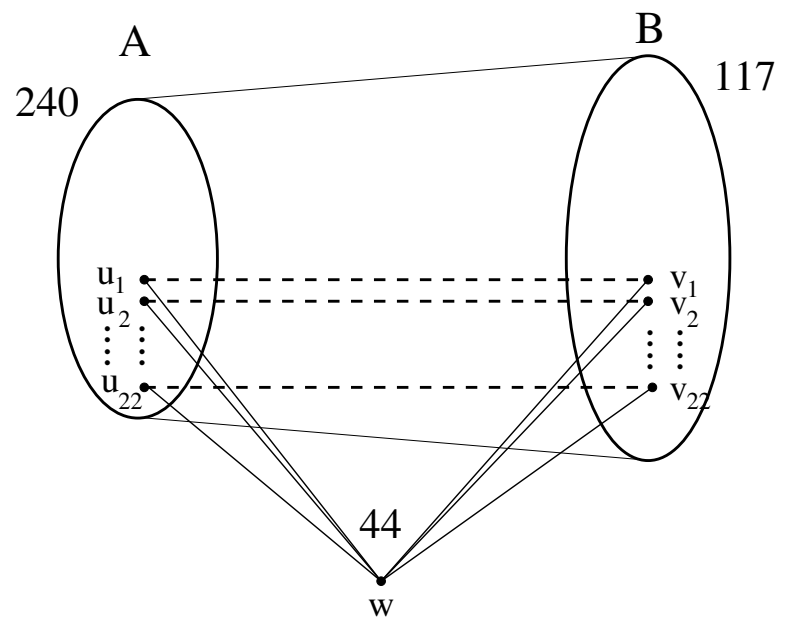

Figure 1: The smallest non-bipartite graph with integer Sombor index.

117 , and 44, respectively. One can easily construct larger such graphs by repeatedly removing edges of another matching of size 22 and connecting their end-vertices to a new vertex, increasing thus the size of class $C$. In that way, we were able to construct graphs with up to 1457 vertices starting from the smallest Euler brick $(44,117,240)$. We have not examined how large (in terms of the number of vertices) graphs can be constructed for a fixed Euler brick $(a, b, c)$.

\section{Concluding remarks}

The non-bipartite graphs constructed in the previous sections are tridegreed and tripartite. It is possible to construct graphs with integer Sombor indices having more than three different degrees. In Figure 2, we show an example with degrees 44, 117, 240, 252 and 275. We start with $K_{117,240}$ and $K_{252,275}$; their classes of bipartition are shown as bold ellipses in Figure 2. From the first one, we remove the edges of a matching of size 22 and proceed to construct $G_{m}$ as in the proof of Theorem 3.1. From the second graph, we remove the edges of a matching of size 119. (The vertices of removed matchings are schematically shown by small ellipses within the classes of bipartition of respective starting graphs.) Add a new vertex and connect it with the end-vertices of removed matching edges; this new vertex, below two ellipses in the right-hand side of Figure 2, is of degree 238. Now, remove edges of a matching of size 2 in the graph on the left and connect their end-vertices in the larger class (now of degree 116) to the vertex of degree 238. Their new degrees become 117 and 240 , respectively. Finally, remove an edge connecting a vertex of degree 252 and a vertex of degree 275 in the right and connect its end-vertices to two vertices of degree 239 in the leftmost ellipse. In that way, we obtain one edge with end-vertices of degrees 240 and 252 and another edge connecting vertices of degrees 240 and 275 . It can be easily checked that all edges of the graph shown in Figure 2 have integer contributions. The degrees of vertices in various classes are indicated in Figure 2 , all other notations are suppressed for clarity. 


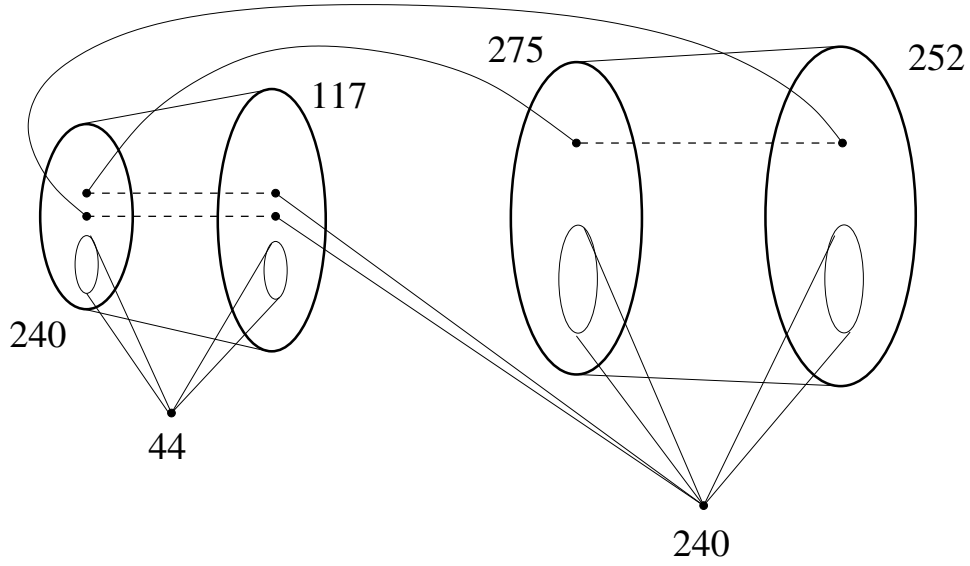

Figure 2: A graph with integer Sombor index and five different degrees.

Crucial for our construction is the fact that 240 appears in two different Euler bricks. Since its multiples, 480, 720, 960,.., appear in an increasing number of Euler bricks, we believe that by iterating the above construction one could obtain nonbipartite graphs with integer Sombor indices and with arbitrarily high number of possible degrees. At the moment, we do not know whether any number of different degrees can be achieved. We leave the details to the interested reader.

\section{Acknowledgments}

Partial support of the Croatian Science Foundation via research project LightMol (Grant no. IP-2016-06-1142) is gratefully acknowledged by T. Došlić. We are grateful to N. Adžaga for performing the search that resulted in the $(44,117,240)$ triple.

\section{References}

[1] H. Abdo, D. Dimitrov, I. Gutman, Graph irregularity and its measures, Appl. Math. Comput. 357 (2019) 317-324.

[2] S. Alikhani, N. Ghanbari, Sombor index of polymers, MATCH Commun. Math. Comput. Chem. 86 (2021) 715-728.

[3] J. A. Bondy, U. S. R. Murty, Graph Theory, Springer, London, 2008.

[4] R. Cruz, I. Gutman, J. Rada, Sombor index of chemical graphs Appl. Math. Comput. 399 (2021) Art\# 126018.

[5] R. Cruz, J. Rada, Extremal values of the Sombor index in unicyclic and bicyclic graphs, J. Math. Chem. 59 (2021) $1098-1116$.

[6] F. Harary, Graph Theory, Addison-Wesley, Reading, 1969.

[7] K. C. Das, A. S. Çevik, I. N. Cangul, Y. Shang, On Sombor Index, Symmetry 13 (2021) Art\# 140.

[8] T. Došlić, I. Martinjak, R. Škrekovski, S. T. Spužević, I. Zubac, Mostar index, J. Math. Chem. 56 (2018) 2995-3013.

[9] X. Fang, L. You, H. Liu, The expected values of Sombor indices in random hexagonal chains, phenylene chains and Sombor indices of some chemical graphs, arXiv:2103.07172 [math.CO], (2021).

[10] I. Gutman, Degree-based topological indices, Croat. Chem. Acta 86 (2013) 351-361.

[11] I. Gutman, Geometric approach to degree-based topological indices: Sombor indices, MATCH Commun. Math. Comput. Chem. 86 (2021) 11-16.

[12] I. Gutman, Some basic properties of Sombor indices, Open J. Discrete Appl. Math. 4 (2021) 1-3.

[13] I. Gutman, A. Dobrynin, The Szeged index - A success story, Graph Theory Notes New York 34 (1998) 37-44.

[14] I. Gutman, B. Furtula, M. B. Ahmadi, S. A. Hosseini, P. S. Nowbandegani, M. Zarrinderakht, The ABC Index Conundrum, Filomat 27 (2013) $1075-1083$.

[15] B. Horoldagva, C. Xu, On Sombor index of graphs, MATCH Commun. Math. Comput. Chem. 86 (2021) 703-713.

[16] M. Juvan, B. Mohar, Bond Contributions to the Wiener Index, J. Chem. Inf. Comput. Sci. 35 (1995) 217-219.

[17] O. Knill, Treasure Hunting Perfect Euler Bricks, Harvard Univ., 2009, http: //abel . math .harvard.edu/ knill/various/eulercuboid/lecture.pdf

[18] V. R. Kulli, I. Gutman, Computation of Sombor indices of certain networks, SSRG Int. J. Appl. Chem. 8 (2021) 1-5.

[19] V. R. Kulli, I. Gutman, (a,b)-KA indices of benzenoid systems and phenylenes: the general case, Int. J. Math. Trends Technol. 67 (2021) 17-20.

[20] X. Li, Y. Shi, A survey on the Randić index, MATCH Commun. Math. Comput. Chem. 59 (2008) 127-156.

[21] Z. Lin, On the spectral radius and energy of the Sombor matrix of graphs, arXiv:2102.03960 [math.CO], (2021).

[22] H. Liu, Ordering chemical graphs by their Sombor indices, arXiv:2103.05995 [math.CO], (2021).

[23] H. Liu, Maximum Sombor index among cacti, arXiv:2103.07924 [math.CO], (2021).

[24] H. Liu, L. You, Z. Tang, J. B. Liu, On the reduced Sombor index and its applications, MATCH Commun. Math. Comput. Chem. 86 (2021) 729-753.

[25] I. Milovanović, E. Milovanović, M. Matejić, On some mathematical properties of Sombor indices, Bull. Int. Math. Virtual Inst. 11 (2021) $341-353$.

[26] M. Randić, On characterization of molecular branching, J. Amer. Chem. Soc. 97 (1975) 6609-6615.

[27] R. L. Rathbun, The Integer Cuboid Table, arXiv:1705.05929v4 [math.NT], (2020).

[28] I. Redžepović, Chemical applicability of Sombor indices, J. Serb. Chem. Soc., DOI: 10.2298/JSC201215006R, In press.

[29] T. Réti, T. Došlić, A. Ali, On the Sombor index of graphs, Contrib. Math. 3 (2021) 11-18.

[30] N. J. A. Sloane, The On-Line Encyclopedia of Integer Sequences, https ://oeis .org

[31] D. Vukičević, M. Gašperov, Bond additive modeling 1. Adriatic indices, Croat. Chem. Acta 83 (2010) 243-260.

[32] Z. Wang, Y. Mao, Y. Li, B. Furtula, On relations between Sombor and other degree-based indices, J. Appl. Math. Comput., DOI: 10.1007/s12190021-01516-x, In press.

[33] H. Wiener, Structural determination of the paraffin boiling points, J. Am. Chem. Soc. 69 (1947) 17-20.

[34] T. Zhou, Z. Lin, L. Miao, The Sombor index of trees and unicyclic graphs with given matching number, arXiv:2103.04645 [math.CO], (2021).

[35] T. Zhou, Z. Lin, L. Miao, The Sombor index of trees and unicyclic graphs with given maximum degree, arXiv:2103.07947 [math.CO], (2021). 